Journal of Soft Computing Paradigm (JSCP) (2020)

Vol.02/ No. 02

Pages: $120-129$

http://irojournals.com/jscp/

DOI: https://doi.org/10.36548/jscp.2020.2.005

\title{
Adaptive Algorithms for Signature Wavelet recognition in the Musical Sounds
}

\author{
Dr. M. Duraipandian, \\ Head of Department, Department of Computer Science and Engineering, \\ Vivekanandha College of Technology for Women, \\ Namakal, India. \\ Email: svsduraipandian@gmail.com
}

\begin{abstract}
The scaling and as well as the wavelet-functions of the wavelet is detected engaging the wavelet-filters that are empowered with the filter-bank principle that is utilized in recognizing the rough calculation and the feature co-efficient of the wavelet-filter. The coefficients recognized by the filter-bank for the musical sounds produced by the musical-instruments enables one to have a signature-wavelet of the sound signal. The signature-wavelet renovates the actual musical signal with insignificant disturbance. In order to recognize the factors (coefficients) the paper employs the least mean square (L-MS), normalized least means square (NL-MS), recursive least square (R-LS) and the QR-Recursive least square (QR-RLS). Among the above four the R-LS and the QR-RLS performs well under all grounds. More over the algorithm converges swiftly compared to the other algorithm. Thus providing an accuracy and SOC (speed of convergence) improved scaling and wavelet-function recognition.
\end{abstract}

Keywords: Signature-Wavelet, Musical Sounds, Adaptive Algorithm, Accuracy, Speed of Convergence

\section{Introduction}

Manifold researches to discover the wavelets closely similar to the signals are laid out to deliver a finest depiction of the signal. Specifically for the signals that are deterministic. There exist two path ways to manage the difficulties in sorting out the wavelet that are similar to the signal. They are

i. Recognizing the fittest similar wavelet for the signal that are under the consideration, from a wavelet set.

ii. Recognizing the similar wavelet utilizing the Adaptive Algorithms.

Every wavelet is rebuilt using its rough estimation and the feature factor calculated using the filter bank principle. The rough set of factors estimated, allows one to have the feature factors to be determined. In many cases the applications that use the wavelet depicts the rough and the feature factors in the form of

ISSN: 2582-2640 (online) 
Journal of Soft Computing Paradigm (JSCP) (2020)

Vol.02/ No. 02

Pages: 120-129

http://irojournals.com/jscp/

DOI: https://doi.org/10.36548/jscp.2020.2.005

quadrature mirror filter (Q-MF) with the mirror image spectrum. These feature factors could be obtained using the conventional or using the adaptive algorithms.

The main aim of the paper is to recognize the signature wavelet of the musical sounds to rebuild the actual signals with minimized insignificant errors. To reconstruct the musical signals the optimal adaptive algorithms is identified in the paper. For this purpose the rough and detailed coefficients of the wavelet are obtained using the various means square error adaptive filter algorithms least mean square and the normalized least means square, recursive-LS, and the QR- Recursive-LS. In the proposed method the filter parameters are repeatedly changed to reduce the error based on the specified criteria. The value determination based on the least mean square and the normalized least mean square requires more computation time and are less accurate compared to the recursive-LS, and the QR- Recursive-LS, so in order to have an accurate and speedy process the algorithm to reconstruct the musical signal is developed based on the recursive-LS, and the QR- Recursive-LS.

The paper is planned with the related works in the section 2, the filter-bank factor detection in section 3, signature wavelet identification in section. 4 , the performance analysis in section 5 and the conclusion in section 6 .

\section{Related Works}

Chapa, Joseph O et al [1] designed algorithms to develop mother-wavelet that are similar to the SOI (signal of interest) these algorithms relied on the closed form solutions to identify the SF-Spectrum from wavelet spectrum such that the results obtained for the similar wavelet are closely resembles the simultaneous solutions that are sampled. Evangelista, et al [2] explores a novel wavelet representation, where the conversion relies on the "pitch synchronous vector" depiction and are adaptable to the oscillatory or the aperiodic attributes of the signals. And compatible for the rate-minimization in coding and synthesis. Gupta, Anubha and Shiv Dutt Joshi, in et al [3] and [4] respectively. The method to present the innovative method for the similar wavelet and the identifying the filter factors and the wavelets for the musical instruments was put forth by the Sinith, M. S., et al [5]

Sharma, et al [6] and [7] examined the MSEAFA on the production of the wavelet and scaling and studied the AFA in detecting the filter factors of the wavelet respectively. Martinek, et al [8] elaborated the "Comparison of the LMS, NLMS, RLS, and QR-RLS algorithms for vehicle noise suppression." FarhangBoroujeny, et al [9] discusses the "Adaptive filters: theory and applications" Jan Zidek et al [10] discusses the use of "The use of lms and rls adaptive algorithms for an adaptive control method of active power filter."

ISSN: 2582-2640 (online) 
Journal of Soft Computing Paradigm (JSCP) (2020)

Vol.02/ No. 02

Pages: 120-129

http://irojournals.com/jscp/

DOI: https://doi.org/10.36548/jscp.2020.2.005

Sharma, et al [11] presets the "novel approach to synthesize sounds of some Indian musical instruments using DWT."

Manoharan, S. et al [12] devised an "A Smart Image Processing Algorithm for Text Recognition, Information Extraction and Vocalization for the Visually Challenged" Kumar, T. Senthil.et al [13] devised an "A Novel Method for HDR Video Encoding, Compression and Quality Evaluation." Manoharan, S. et al [14] elaborated the "Study on Hermitian Graph Wavelets in Feature Detection" .Raj, J. S., et al [15] proposed a "Recurrent Neural Networks and Nonlinear Prediction in Support Vector Machines."

\section{Filter-Bank Factor detection using the Adaptive Algorithms}

All the four algorithms, least-Mean square, normalized least- mean square, recursive- least square and the QR-recursive least square is implemented to estimate the factors of the filter bank for the wavelet "DB3" Choosing the step dimension are very difficult as stated in [5-8] the dimension of the steps chosen with more than .17 would result in no convergence making the wavelet reconstruction impossible. The values obtained by Least-Mean Square are displayed in the table.1 below. The similar set up is done for the estimating the filter factor using the normalized least- mean square this algorithm is insensitive of step size. The recursive-least square algorithm and the QR-Recursive-least square is utilized for estimating the factors, the experiment showed that the factor obtained by the recursive-least square algorithm and the QRRecursive-least square were very much similar to the expected values and were capable enough of performing reconstruction of the wavelets with the speedy convergence. All the filter bank factors observed the number of iterations and the dimension of the step for every algorithm is stated below in table. 1

ISSN: 2582-2640 (online) 
Journal of Soft Computing Paradigm (JSCP) (2020)

Vol.02/ No. 02

Pages: $120-129$

http://irojournals.com/jscp/

DOI: https://doi.org/10.36548/jscp.2020.2.005

\begin{tabular}{|c|c|c|c|c|c|}
\hline $\begin{array}{l}\text { Actual } \\
\text { Factors Of } \\
\text { Filter } \\
\text { Bank }\end{array}$ & $\begin{array}{l}\text { Dimension } \\
\text { of Steps }\end{array}$ & $\begin{array}{l}\text { Filter } \\
\text { Factors of } \\
\text { L-MS }\end{array}$ & $\begin{array}{l}\text { Filter } \\
\text { Factors of } \\
\text { NL-MS }\end{array}$ & $\begin{array}{l}\text { Filter } \\
\text { Factors of } \\
\text { R-LS }\end{array}$ & $\begin{array}{l}\text { Filter } \\
\text { Factor of } \\
\text { QR-R-LS }\end{array}$ \\
\hline \multirow[t]{5}{*}{$\begin{array}{l}{[0.0499} \\
-0.1209 \\
-0.1910 \\
0.6505 \\
1.1412 \\
0.4706]\end{array}$} & 0.011 & $\begin{array}{l}{[0.0474,} \\
-0.1170, \\
-0.1900, \\
0.6406, \\
1.1434, \\
0.4650]\end{array}$ & $\begin{array}{l}{[0.0458,} \\
-0.1223, \\
-0.1920, \\
0.6151,1.0 \\
563, \\
0.4235]\end{array}$ & $\begin{array}{l}\text { [0.0497, } \\
-0.1246, \\
-0.1876, \\
0.6486, \\
1.1416, \\
0.4704]\end{array}$ & $\begin{array}{l}{[0.0498,} \\
-0.1247, \\
-0.1877, \\
0.6485, \\
1.1414, \\
0.47045\end{array}$ \\
\hline & .055 & $\begin{array}{l}{[0.0187,} \\
-0.1200, \\
-0.2222, \\
0.6318, \\
1.1473, \\
0.5000]\end{array}$ & $\begin{array}{l}{[0.0457,} \\
-0.1243, \\
-0.1922, \\
0.6443, \\
1.1323, \\
0.4703]\end{array}$ & $\begin{array}{l}{[0.0463,} \\
-0.1249, \\
-0.1958, \\
0.6451 \\
1.1401 \\
0.4689]\end{array}$ & $\begin{array}{l}{[0.0464,} \\
-0.1248, \\
-0.1957, \\
0.6452, \\
1.1402, \\
0.4688]\end{array}$ \\
\hline & .0100 & $\begin{array}{l}{[-0.3811,} \\
-0.1851, \\
-0.1950, \\
0.5448, \\
0.685, \\
0.0337]\end{array}$ & $\begin{array}{l}{[0.0432,} \\
-0.1324, \\
-0.1947, \\
0.6458, \\
1.1450, \\
0.4653]\end{array}$ & $\begin{array}{l}{[0.0470,} \\
-0.1244, \\
-0.1917, \\
0.6478, \\
1.1363 \\
0.4690]\end{array}$ & $\begin{array}{l}{[0.0470,} \\
-0.1244, \\
-0.1918, \\
0.6478, \\
1.1363, \\
0.4690]\end{array}$ \\
\hline & .015 & $\begin{array}{l}{[-0.6961,} \\
1.2182, \\
-0.03197, \\
-1.6593, \\
1.9380, \\
0.0424]\end{array}$ & $\begin{array}{l}{[0.0456,} \\
-0.1050, \\
-0.1678, \\
0.6512, \\
1.1340, \\
0.4824]\end{array}$ & $\begin{array}{l}\text { [0.0497, } \\
-0.1230, \\
-0.1910, \\
0.6488, \\
1.1442, \\
0.4709]\end{array}$ & $\begin{array}{l}{[0.0497,} \\
-0.1234, \\
-0.1910, \\
0.6488, \\
1.1443, \\
0.4709]\end{array}$ \\
\hline & .30 & $\begin{array}{l}{[-1.013,} \\
48.713, \\
2.9040, \\
-1.6613, \\
-1.1017, \\
1.0041] \times \\
10200\end{array}$ & $\begin{array}{l}{[0.0310,} \\
-0.1220, \\
-0.1345, \\
0.6534, \\
1.1793, \\
0.4834]\end{array}$ & $\begin{array}{l}{[0.0461,} \\
-0.1226, \\
-0.1988, \\
0.6445 \\
1.1374 \\
0.4682]\end{array}$ & $\begin{array}{l}{[0.0461,} \\
-0.1227, \\
-0.1988, \\
0.6446, \\
1.1375, \\
0.4684]\end{array}$ \\
\hline $\begin{array}{l}\text { Iteration } \\
\text { count }\end{array}$ & & $4 n$ & $2 n$ & $\mathrm{n}$ & $\mathrm{n}$ \\
\hline
\end{tabular}

Table.1 Filter Factors Acquired from L-MS, NL-MS, R-LS and QR-R-LS

The ' $n$ ' denotes the filter bank factor count and the iteration are closely associated with the factors, the changes in the repetition durations causes severe variations in the count of the factors. The "mean square error" attained by the recursive-least square and the QR-recursive- Least square is minimum and converges swiftly compared to the other two.

ISSN: 2582-2640 (online) 
Journal of Soft Computing Paradigm (JSCP) (2020)

Vol.02/ No. 02

Pages: $120-129$

http://irojournals.com/jscp/

DOI: https://doi.org/10.36548/jscp.2020.2.005

\section{Proposed Algorithm combining R-LS and the QR-recursive-LS for SWR in Musical Sounds}

\section{Recursive-Least Square:}

The figure. 1 is the flow diagram of the scaling function the steps in the R-LS to get the factors of the filter initiates with the estimation of the filter output followed by calculating the error, and updating the factors.

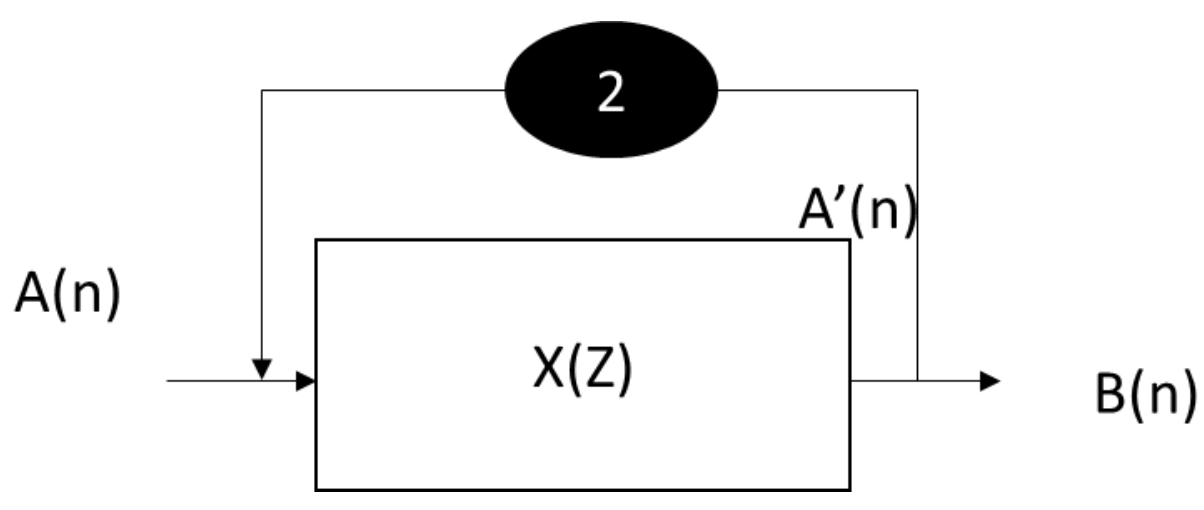

Figure.1 Scaling Function

Later the gain vectors and the inverse correlation matrix are determined. The R-LS though delivers a speedy convergence. "However when the inverse correlation matrix loses the Hermitian symmetry it deviates, this limits the application of the algorithm", the QR- recursive-LS solves this problem as it does the decomposition of the QR straight on the input signals correlation matrix. So the method utilized in the paper to recognize the signature wavelet for the musical sounds combines both the Recursive-LS and the QR recursive-Least square [8]. To detect the factors of the filter bank for the musical sounds. These factors could be utilized in producing the wavelet functions and the scaling for the musical sounds. The algorithm is as follows.

I. The musical signals are taken in the .wav format.

II. The sampling rate is chosen and the partial signal is examined based on the rate of the sampling.

III. Decide the filter order, smooth wavelet functions are achieved only for the filters of larger order.

IV. Call the Recursive-least square and the QR-recursive-least square algorithm.

V. Determine the error once the iteration is completed. IF the iteration are have reached the limit specified then STOP the procedure of convergence, ELSE GO TO III 
Journal of Soft Computing Paradigm (JSCP) (2020)

Vol.02/ No. 02

Pages: 120-129

http://irojournals.com/jscp/

DOI: https://doi.org/10.36548/jscp.2020.2.005

VI. On satisfying the benchmarks for the error reserve the factors achieved.

VII. Utilize the filter factors to develop the wavelet and the scaling function.

\section{Performance Analysis}

The designed algorithm is tested by applying the algorithm to various musical sounds recorded from various musical gadgets. As mentioned the in the algorithm above the signal as are collected in the .wav format and the subjected to sampling $45.2 \mathrm{~K}$ samples, every portion of music gathered from each instrument is different from each other. The signal size for a string gadget is $10 \mathrm{~ms}$ and the keyboard is $100 \mathrm{~ms}$ etc. the filter order is set to 33 so that the acquired wavelet would be smooth. The total value factor achieved for each instrument is listed below in table. 2

\begin{tabular}{ll}
$\begin{array}{ll}\text { Musical } \\
\text { Sounds }\end{array}$ & Filter Factors \\
\hline Keyboard & {$[0.01930 .01630 .00620 .0041-0.0010-0.0069-0.0035-0.0075-0.0158$} \\
& $-0.0166-0.0134-0.0154-0.0176-0.0205-0.0170-0.0087-0.0087$ \\
& $-0.0089-0.0131-0.0086-0.0103-0.0068-0.0041-0.0005-0.0089$ \\
& $-0.0107-0.0084-0.00430 .0016-0.0013-0.0043-0.0056]$ \\
Guitar & {$[0.0113-0.0021-0.0057-0.0066-0.0062-0.0017-0.0076-0.0104$} \\
& $-0.0131-0.0173-0.0236-0.0299-0.0283-0.0240-0.0195-0.0192$ \\
& $-0.0177-0.0158-0.0166-0.0144-0.0173-0.0203-0.0180-0.0088$ \\
\hline Bongos & $-0.0011-0.00030 .00370 .00250 .00420 .00510 .00260 .0059]$ \\
& {$[0.03020 .03110 .02440 .02340 .02030 .02090 .02430 .02550 .02560 .0289$} \\
& 0.02910 .02950 .02840 .03300 .02880 .02630 .02360 .03020 .02360 .0220 \\
Flute & 0.02580 .01890 .01640 .01290 .01560 .01410 .01500 .01450 .01430 .0173 \\
& $0.01630 .0143]$ \\
& {$[0.07130 .03750 .02890 .01130 .01060 .0021-0.0259-0.0038-0.0297$} \\
& $-0.0368-0.0282-0.0371-0.0430-0.0342-0.0438-0.0514-0.0645$ \\
& $-0.0543-0.0576-0.0623-0.0595-0.0495-0.0629-0.0522-0.0493$ \\
\hline & $-0.0488-0.0397-0.0517-0.0383-0.0151-0.0064-0.0135]$ \\
\hline
\end{tabular}

Table.2 Filter Factors Achieved for Key Board, Guitar, Bongos and Flute

The wavelet function as well as the scaling obtained for the various instrument mentioned above is acquired using the QR-recursive-LS combined with Recursive-LS, is shown below in figure 2(a), 2(b), 2(c) and2 (d). Utilizing the scaling and the wavelet-function to blend the musical sounds enables one to have a better and improved blending resembling the actual notes. 
Journal of Soft Computing Paradigm (JSCP) (2020)

Vol.02/ No. 02

Pages: $120-129$

http://irojournals.com/jscp/

DOI: https://doi.org/10.36548/jscp.2020.2.005
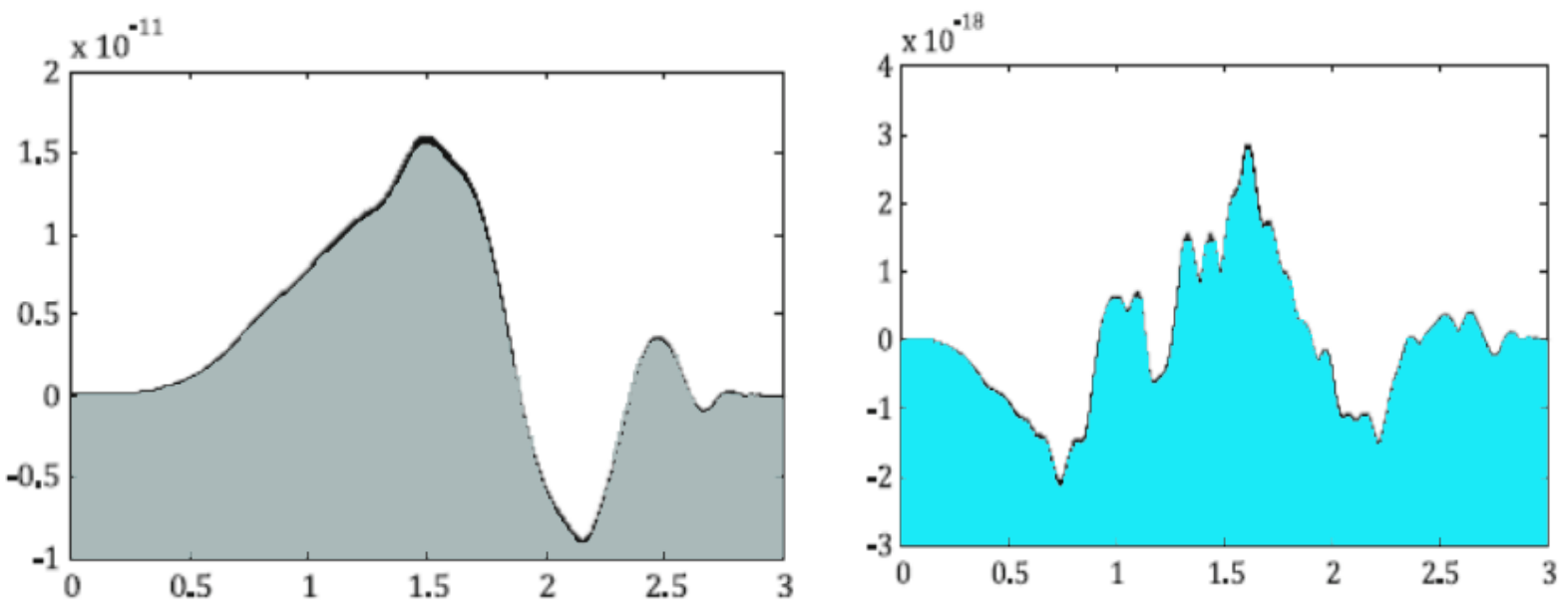

Figure. 2(a) Keyboard Scaling and Wavelet
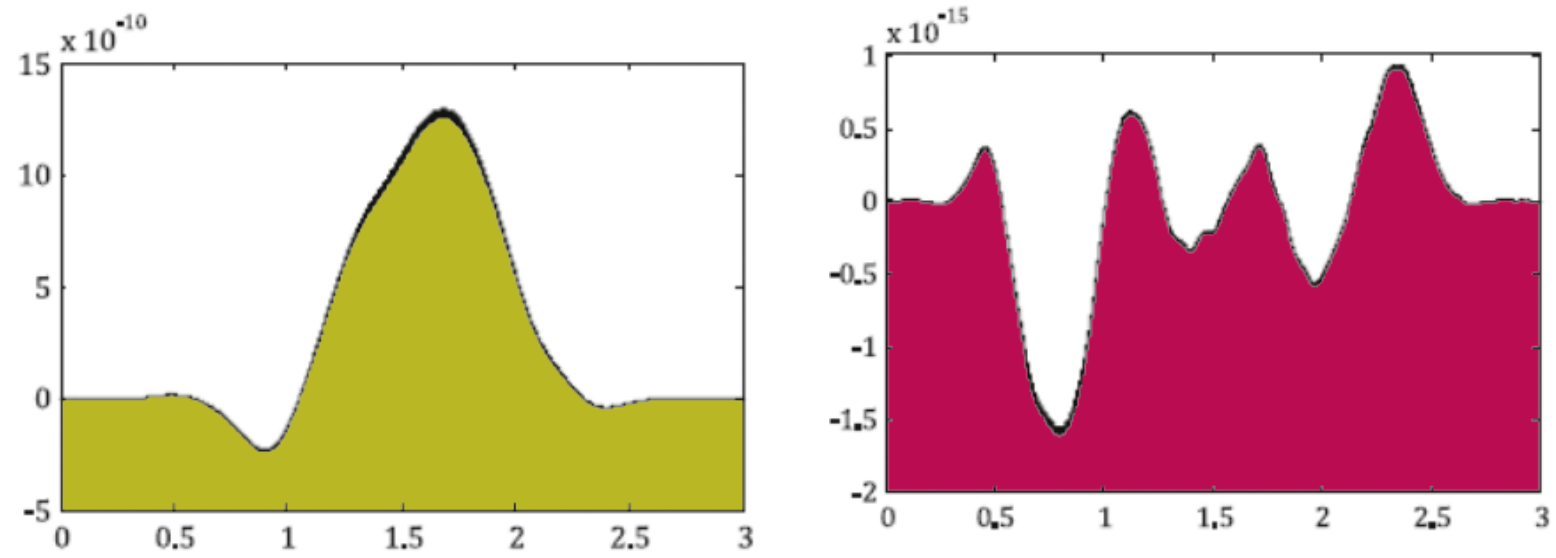

Figure. 2(b) Guitar Scaling and Wavelet 
Journal of Soft Computing Paradigm (JSCP) (2020)

Vol.02/ No. 02

Pages: 120-129

http://irojournals.com/jscp/

DOI: https://doi.org/10.36548/jscp.2020.2.005
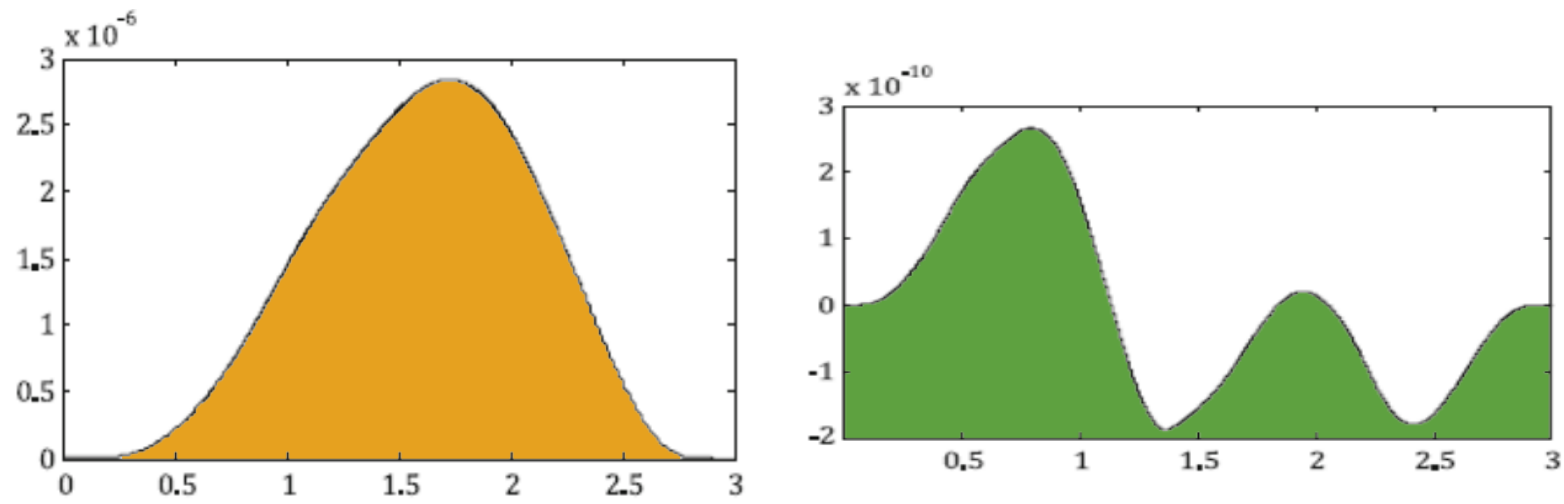

Figure 2(c) Bongos Scaling and Wavelet
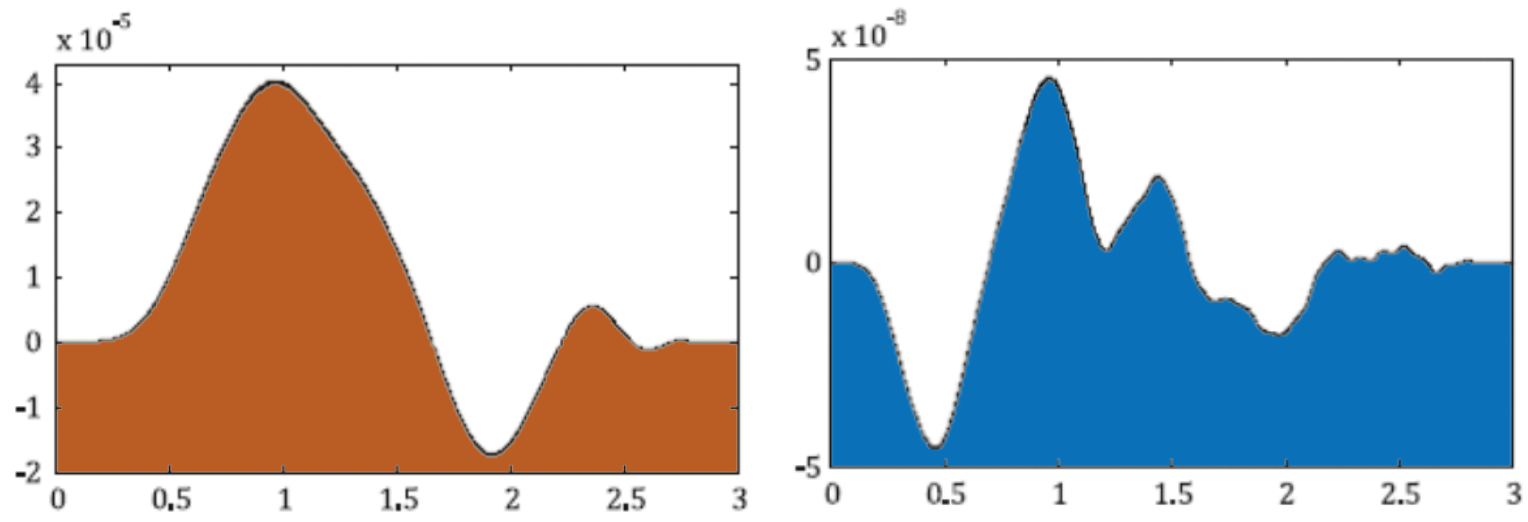

Figure 2(d) Flute Scaling and Wavelet

\section{Conclusion}

The paper recognizes the factors of the filter-bank for the musical sounds using the recursive- least square and the QR-Recursive-least Square through the obtained factors the wavelet-function and the scaling are contrived. The four adaptive procedures were used to acquire the factors of the filter-bank but the QR-RLS and the R-LS combined procedure offered closer values compared to the other procedure this was explained clearly through the table.1 so the paper utilized the QR-R-LS and the R-LS combined procedure to develop an algorithm to detect the musical sounds filter bank factor and plot the scaling and the waveletfunction using the acquired factors. The procedure put forth was tested with the instruments such as key 
Journal of Soft Computing Paradigm (JSCP) (2020)

Vol.02/ No. 02

Pages: 120-129

http://irojournals.com/jscp/

DOI: https://doi.org/10.36548/jscp.2020.2.005

board, flute, bongos and guitar. The results observed in the table.2 and the figures 2 (a, b, c, d) proved the improved accuracy achieved in the minimum time duration using the QR-R-LS combined with the R-LS.

\section{References}

[1] Chapa, Joseph O., and Raghuveer M. Rao. "Algorithms for designing wavelets to match a specified signal." IEEE transactions on signal processing 48, no. 12 (2000): 3395-3406.

[2] Evangelista, Gianpaolo. "Pitch-synchronous wavelet representations of speech and music signals." IEEE transactions on signal processing 41, no. 12 (1993): 3313-3330.

[3] Gupta, Anubha, S. D. Joshi, and Surendra Prasad. "On a new approach for estimating wavelet matched to signal." In Proceedings of the Eighth National Conference on Communications, Bombay. 2002.

[4] Gupta, Anubha, Shiv Dutt Joshi, and Surendra Prasad. "A new approach for estimation of statistically matched wavelet." IEEE transactions on signal processing 53, no. 5 (2005): 17781793.

[5] Sinith, M. S., Madhavi Nisha Nair, Niveditha P. Nair, and S. Parvathy. "Identification of wavelets and filter bank coefficients in musical instruments." In 2010 International Conference on Audio, Language and Image Processing, pp. 727-731. IEEE, 2010.

[6] Sharma, R. A. G. H. A. V. E. N. D. R. A., and V. PREM Pyara. "A comparative analysis of mean square error adaptive filter algorithms for generation of modified scaling and wavelet function." Int J Eng Sci Technol 4, no. 4 (2012): 1396-1401.

[7] Sharma, Raghavendra, and V. Prem Pyara. "Comparative study of adaptive algorithms for identification of filter bank coefficients of wavelets." Proceedings, international journal of computer applications ${ }^{\circledR}(I J C A)$ (2012): 21-25.

[8] Martinek, Radek, Radana Kahankova, Jan Nedoma, Marcel Fajkus, and Michal Skacel. "Comparison of the LMS, NLMS, RLS, and QR-RLS algorithms for vehicle noise suppression." In Proceedings of the 10th International Conference on Computer Modeling and Simulation, pp. 23-27. 2018.

[9] Farhang-Boroujeny, Behrouz. Adaptive filters: theory and applications. John Wiley \& Sons, 2013.

[10] Martinek, Radek, Jan Zidek, Petr Bilik, Jakub Manas, Jiri Koziorek, Zhaosheng Teng, and He Wen. "The use of lms and rls adaptive algorithms for an adaptive control method of active power filter." Energy and Power Engineering 5, no. 04 (2013): 1126.

[11] Sharma, Raghavendra, and V. Prem Pyara. "A novel approach to synthesize sounds of some Indian musical instruments using DWT." International Journal of Computer Applications 45, no. 13 (2012): 19-22. 
Journal of Soft Computing Paradigm (JSCP) (2020)

Vol.02/ No. 02

Pages: 120-129

http://irojournals.com/jscp/

DOI: https://doi.org/10.36548/jscp.2020.2.005

[12] Manoharan, S. (2019). A Smart Image Processing Algorithm for Text Recognition, Information Extraction and Vocalization for the Visually Challenged. Journal of Innovative Image Processing (JIIP), 1(01), 31-38.

[13] Kumar, T. Senthil. "A Novel Method for HDR Video Encoding, Compression and Quality Evaluation." Journal of Innovative Image Processing (JIIP) 1, no. 02 (2019): 71-80.

[14] Manoharan, S. (2019). Study On Hermitian Graph Wavelets in Feature Detection. Journal of Soft Computing Paradigm (JSCP), 1(01), 24-32.

[15] .Raj, J. S., \& Ananthi, J. V. (2019). Recurrent Neural Networks and Nonlinear Prediction in Support Vector Machines. Journal of Soft Computing Paradigm (JSCP), 1(01), 33-40.

\section{Authors Biography}

Dr. M. Duraipandian is the Head of Department, in Department of Computer Science and Engineering, in Vivekanandha College of Technology for Women, Namakal, India. His interested area of research includes the Fuzzy Computing, Evolutionary Computing, Data Mining, Artificial Intelligence, Evolutionary Computing, Neural Networks, Autonomous Techniques, Decision Support and Pattern Recognition 